\title{
Epidemic spreading on metapopulation networks including migration and
} demographics

\author{
Yongwang Gong, ${ }^{1,2,}$, Michael Small ${ }^{2,3}$ \\ 1 School of Information Engineering, Yancheng Institute of Technology, Yancheng 224051, China \\ 2 School of Mathematics and Statistics, The University of Western Australia, Perth 6009, Australia \\ 3 Mineral Resources, CSIRO, Kensington, 6151, Australia \\ *Corresponding author; Email address: gong_yw@126.com
}

\begin{abstract}
Epidemic dynamics in a structured population has been widely investigated in recent years by utilizing the metapopulation framework with a reaction-diffusion approach. In this paper, we study epidemic spreading on metapopulation networks including migration and demographics, wherein population dynamics in each node (a patch) follows the logistic model with a heterogeneous capacity. The epidemic threshold is theoretically calculated at a meanfield level and is then evaluated by Monte Carlo simulations. It is shown that heterogeneity of capacity drastically decreases the threshold, and conversely increasing the migration rate slightly increases the threshold. Interestingly, we observe Monte Carlo simulations showing the effect of heterogeneity of capacity and migration on the epidemic prevalence above the epidemic threshold. Heterogeneity of capacity enhances epidemic spreading in the initial stage but has no impact on the final infection density. The migration rate has pronounced impact on both temporal spreading behaviour and endemic state.
\end{abstract}

Keywords: Epidemic spreading, Metapopulation network, Reaction-diffusion approach, Migration, Demographics

Epidemic spreading on metapopulation networks has attracted attention in recent years, wherein each node represents a patch consisting of any number of individuals and edges denote pathways for individual migration. Previous studies have shown that network structure, migration pattern, and local properties have an essential impact on epidemic spreading. This paper proposes a new epidemic model on metapopulation networks by incorporating demographic and migration simultaneously to study how and to what degree population dynamics and individual migration influence epidemic spreading. In this model, population dynamics in each node (a patch) follows the logistic model with a heterogeneous capacity and individual migration follows a random walk pattern. By theoretical analysis and Monte Carlo simulations, we find that the heterogeneity of capacity and migration rate play a complicated role in epidemic threshold, as well as temporary epidemic behaviours and final infection density above the epidemic threshold. 


\section{INTRODUCTION}

The study of epidemic spreading on complex networks is important to our understanding of infectious diseases dynamics in a population and computer virus/worms transmission on the Internet as well as to further develop diseases control strategies. Toward this end, many works have been carried out to investigate epidemic spreading using various theoretical models and methods by researches from different disciplines [1-6].

In recent years, the spread of an infectious disease in a spatially structured population with the migration of individuals has begun to attract attention. An effective theoretical framework, called metapopulation framework with a reaction-diffusion approach, has been introduced to study such epidemic dynamics [7-8]. In this framework, the structured population is encoded as a metapopulation network in which each node represents a patch consisting of any number of individuals and links connecting different patches denote routes for individuals' migration. Epidemic dynamics (reaction) occurs inside each patch ruled by a standard compartmental model (either SIS [3] or SIR [9]), and then a migration process (diffusion) of individuals happens among neighbouring patches. The migration of infected individuals to unaffected patches possible results in epidemic spreading globally over the system.

By using the metapopulation framework and reaction-diffusion approaches, these studies have shown that many factors such as network topology [7-8], mobility patterns [10-11], path choices [11], dispersal distance [12] and local properties, such as city-size heterogeneity [13], local population structure [14-15], local heterogeneous mixing [16], and heterogeneous infection rates [17], have a significant influence on epidemic dynamics in a metapopulation. For instance, the heterogeneity of network topology can decrease the global invasion threshold [7], and individuals travel avoiding locations with high levels of infection prevalence instead enhance disease transmission [11]. In contrast, Saldaña et al. consider reaction and diffusion processes to occur simultaneously [18], which turns out to be the correct assumption for a suitable continuous-time formulation of metapopulation models for epidemic spreading [19- 20].

In previous studies, epidemic models are formulated based on a constant population size of a system. This is reasonable for describing the situation of an acute disease spreading. However, in some cases, the population is growing or decreasing significantly because of fatal disease and other factors [21-25], such that epidemic models have to consider the variation in the number of individuals. As far as we know, there are few epidemic models on metapopulation networks considering simultaneously of both migration and demographics apart from two literatures [26-27]. In [26], the authors studied the dynamics of a specific 
disease, i.e., tuberculosis, in a heterogeneous metapopulation by using SEI model with a constant recruitment. In [27], the asymptotical stabilities of two SIS models in metapopulation networks were analysed. One model has a constant recruitment and the other has logistic recruitment such that the capacity of each node is homogeneous.

Here we present a general theoretical framework based on the continuous-time reactiondiffusion approach to study the epidemic spreading in a spatially structured population with migration and demographics. Different from Refs. [26-27], in our theoretical framework, a SIR model is utilized and population dynamics in each patch follows the logistic growth model with a heterogeneous capacity. Moreover, we mainly focus on the joint impact of the heterogeneity of capacity and migration on epidemic spreading and take Monte Carlo simulation method, not a numerical simulation, to validate the theoretical findings. The theoretical threshold is derived and it is shown that the heterogeneity of capacity of patches drastically decreases the threshold, and instead increase of the migration rate increases the threshold slightly. It is also shown by simulations that the heterogeneity of capacity enhances epidemic spreading in the initial stage but has no impact on the final infection density if considering the same final population size. The migration rate has pronounced impact on both temporal spreading behaviours and endemic state.

The paper is organized as follows. In section 2, we present an epidemic model on metapopulation networks with migration, and population dynamics in each node (patch) following the logistic model with a heterogeneous capacity. We then analyse theoretically the model and derive an expression of threshold in term of the average population capacity in section 3. Section 4 performs extensively Monte Carlo simulations to validate the theoretical findings and further demonstrate epidemic prevalence above the threshold. Finally we conclude and discuss our work in Section 5.

\section{MODEL FORMULATION}

In this paper, we consider epidemic dynamics on $V$ spatially structured interacting patches connected by migration of individuals that can be encoded as a scale-free metapopulation network with a power-law degree distribution $P(k) \sim k^{-\gamma}$. Inside each patch $i$, a classical SIR epidemic model is adopted in which the individuals are divided into three types: susceptible $(\mathrm{S})$, infected (I) and recovered (R) individuals. If assume that infection and recover parameters are respectively $\beta$ and $\mu$, the infection process can be schematically represented by $I+S \stackrel{\beta}{\rightarrow} 2 I$ and $I \stackrel{\mu}{\rightarrow} R$. At the same time, we assume the population dynamics in each patch 
follows the logistic growth model [24, 28] with a heterogeneous capacity, and individuals migrate randomly among neighbouring patches.

Let $\rho_{S, i}(t), \rho_{I, i}(t)$ and $\rho_{R, i}(t)$ denote the number of susceptible, infected and recovered individuals in patch $i$ at time $t$, respectively. Hence $\rho_{i}(t)=\rho_{S, i}(t)+\rho_{I, i}(t)+\rho_{R, i}(t)$ is the total number of individuals in patch $i$ at time $t$. We represent the natural birth and death rates by $b$ and $d$ respectively with $\eta=b-d$ denoting the growth rate. Define the real birth rate as density-dependent $b-\eta \rho_{i}(t) / \Theta_{i}$, and the real death rate as density-independent $d$, where $\Theta_{i}$ is the capacity of population in patch $i$, and assume all newborns are susceptible. Thus, we have the infection dynamics equation with logistic growth population in patch $i$ at mean-filed level without migration

$$
\begin{aligned}
& \frac{d \rho_{S, i}(t)}{d t}=\left(b-\eta \rho_{i}(t) / \Theta_{i}\right) \rho_{i}(t)-\beta \rho_{S, i}(t) \rho_{I, i}(t)-d \rho_{S, i}(t), \\
& \frac{d \rho_{I, i}(t)}{d t}=\beta \rho_{S, i}(t) \rho_{I, i}(t)-\mu \rho_{I, i}(t)-d \rho_{I, i}(t), \\
& \frac{d \rho_{R, i}(t)}{d t}=\mu \rho_{I, i}(t)-d \rho_{R, i}(t) .
\end{aligned}
$$

Note that by adding the three sub-equations in Eq. (1), we will get the logistic equation $d \rho_{i}(t) / d t=\eta \rho_{i}(t)\left(1-\rho_{i}(t) / \Theta_{i}\right)$ meaning that demographic dynamics in each patch strictly follows logistic growth.

At the patch level, due to the distinct local properties of each patch such as size, resources and centrality, we define a heterogeneous capacity for different patches

$$
\Theta_{i}=\frac{k_{i}^{\alpha}}{\left\langle k^{\alpha}\right\rangle} \bar{\Theta}, 0 \leq \alpha \leq 2,
$$

where $k_{i}$ is the degree of node (patch) $i$, and $\bar{\Theta}=\sum_{i} \Theta_{i} / V$ is the average capacity of all patches in the system. Parameter $\alpha$ is a tunable exponent that governs the heterogeneous level of the capacity. Here we assume that the larger degree patches have a greater capacity $(\alpha \geq 0)$ owing to the fact that the hub patches (such as important cities) in real society generally have more resources to support more people's livelihoods, and at the same time, we also limit $0 \leq \alpha \leq 2$, because it is unrealistic to impose more extreme fluctuations of capacity between different patches.

In the following, we model the migration of individuals between connected patches. For simplicity and mainly focus on the impact of demographic factors on the epidemic, we use the stochastic migration model (i.e., each individual selects randomly one of the neighbour patches as its destination) with a homogeneous rate $p$. Thus, individuals in each patch $i$ with degree $k_{i}$ will move to one of their neighbouring patch with probability $p / k_{i}$. The schematic representation of infection dynamics with demographic and migration dynamics is shown in 
Fig. 1. For the convenience of readers, all parameters used in these two dynamics processes are reported in Table 1

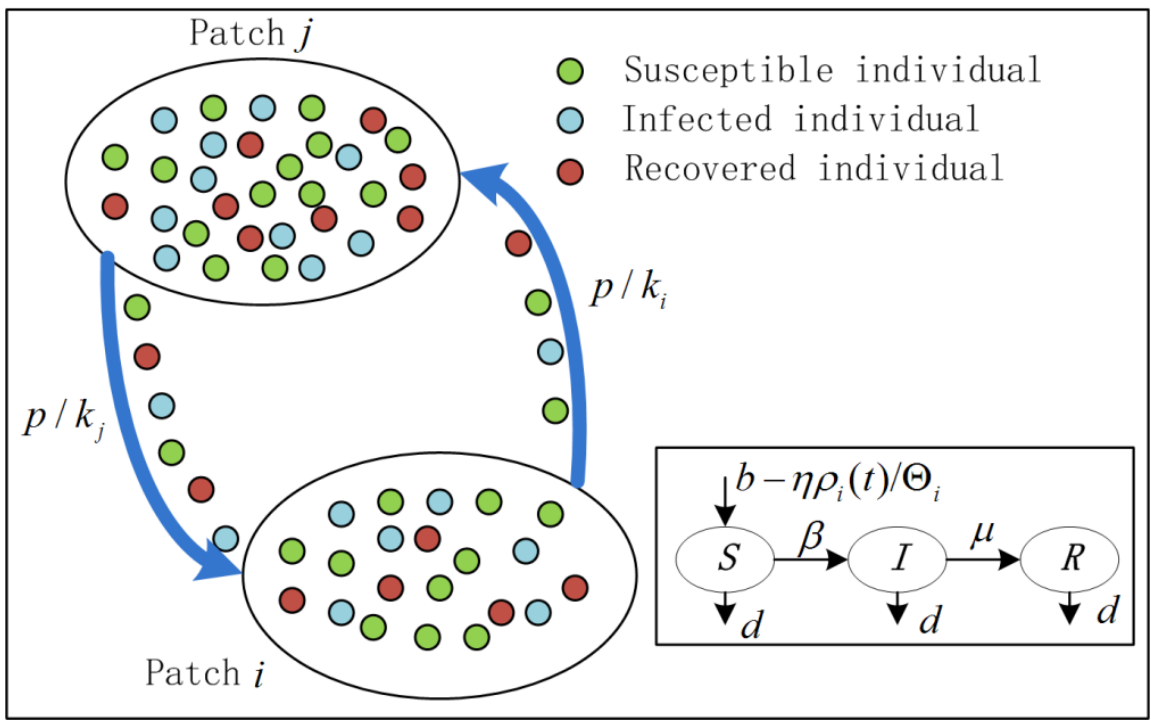

Fig. 1 Schematic representation of our SIR model in a metapopulation network with demographic. In this model, it contains migration process of individuals with a homogenous migration rate $p$ (thus $p / k_{i}$ is migration rate along any given link of patch $i$ ) and epidemic spreading process in each patch with demographics following logistic growth.

Table 1 Dynamics parameters

\begin{tabular}{l|l|l}
\hline \hline Parameter & Meaning & Range \\
\hline$\beta$ & infection rate & {$\left[\begin{array}{ll}0 & 1\end{array}\right]$} \\
\hline$\mu$ & recover rate & {$\left[\begin{array}{ll}0 & 1\end{array}\right]$} \\
\hline$b$ & natural birth rate & {$\left[\begin{array}{ll}0 & 1\end{array}\right]$} \\
\hline$d$ & natural death rate & {$\left[\begin{array}{ll}0 & 1\end{array}\right]$} \\
\hline$\eta=b-d$ & growth rate & {$\left[\begin{array}{ll}0 & 1\end{array}\right]$} \\
\hline$p$ & migration rate & {$\left[\begin{array}{ll}0 & 1\end{array}\right]$} \\
\hline \hline
\end{tabular}

By incorporating the migration process into Eq. (1), we formulate a modified SIR epidemic model on a metapopulation network with migration between patches. Based on the assumption that the patches with the same degree are statistically equivalent [8], we define degree-block variables $\rho_{S, k}(t), \rho_{I, k}(t)$ and $\rho_{R, k}(t)$ denoting the average number of susceptible, infected and recovered individuals in patches with degree $k$ at time $t$. Then the average population size of each patch with degree $k$ at time $t$ is given by

$$
\rho_{k}(t)=\rho_{S, k}(t)+\rho_{I, k}(t)+\rho_{R, k}(t) .
$$

The dynamics of the system at the patch level with mean-field approximation is given as follows 


$$
\begin{aligned}
\frac{d \rho_{S, k}(t)}{d t}= & \left(b-\frac{\eta \rho_{k}(t)}{\theta_{k}}\right) \rho_{k}(t)-\beta \rho_{S, k}(t) \rho_{I, k}(t)-d \rho_{S, k}(t)-p \rho_{S, k}(t) \\
& +k \sum_{k^{\prime}} P\left(k^{\prime} / k\right) \frac{p}{k^{\prime}} \rho_{S, k^{\prime}}(t), \\
\frac{d \rho_{I, k}(t)}{d t}= & \beta \rho_{S, k}(t) \rho_{I, k}(t)-\mu \rho_{I, k}(t)-d \rho_{I, k}(t)-p \rho_{I, k}(t)+k \sum_{k^{\prime}} P\left(k^{\prime} / k\right) \frac{p}{k^{\prime}} \rho_{I, k^{\prime}}(t), \\
\frac{d \rho_{R, k}(t)}{d t}= & \mu \rho_{I, k}(t)-d \rho_{R, k}(t)-p \rho_{R, k}(t)+k \sum_{k^{\prime}} P\left(k^{\prime} / k\right) \frac{p}{k^{\prime}} \rho_{R, k^{\prime}}(t), \\
\frac{d \rho_{k}(t)}{d t}= & \eta\left(1-\frac{\rho_{k}(t)}{\Theta_{k}}\right) \rho_{k}(t)-p \rho_{k}(t)+k \sum_{k^{\prime}} P\left(k^{\prime} / k\right) \frac{p}{k^{\prime}} \rho_{k^{\prime}}(t)
\end{aligned}
$$

where $k_{\min } \leq k \leq k_{\max }$ is the degree of the patches with $k_{\min }$ and $k_{\max }$ being the minimum and maximum degrees respectively, $\Theta_{k}=\frac{k^{\alpha}}{\left\langle k^{\alpha}\right\rangle} \bar{\Theta}$ (i.e., the corresponding block-degree variable of $\Theta_{i}$ ) stands for the average capacity of patches with degree $k$, and $P\left(k^{\prime} / k\right)$ indicates the condition probability that a patch with degree $k$ connects to a patch with degree $k^{\prime}$. The first three sub-equations describe the dynamics of the susceptible, infected, recovered individuals, and the last sub-equation characterises the population demographics. The first three terms on the right-hand side of Eq. (4a) account for the variation in the number of susceptible individuals due to infection dynamics and demographics within patches. The fourth and fifth terms represent, respectively, the number of outgoing and incoming susceptible individuals due to migration. In the same way, it is easy to understand the meaning of terms of Eq. (4b) and Eq. (4c) for infected and recovered individuals. Demographics of Eq. (4d) consists of two processes-the classical logistic growth, and, population variation resulting from the migration.

For an uncorrelated metapopulation network, we have $P\left(k^{\prime} / k\right)=k^{\prime} P\left(k^{\prime}\right) /\langle k\rangle[29]$ where $\langle k\rangle=\sum_{k} k P(k)$ is the average degree of the network and $P(k)$ is the degree distribution of the network. By substituting this equation into Eq. (4) and then performing a simple algebraic calculation, this becomes

$$
\begin{aligned}
& \frac{d \rho_{S, k}(t)}{d t}=\left(b-\frac{\eta \rho_{k}(t)}{\theta_{k}}\right) \rho_{k}(t)-\beta \rho_{S, k}(t) \rho_{I, k}(t)-d \rho_{S, k}(t)-p \rho_{S, k}(t)+p \frac{k}{\langle k\rangle} \overline{\rho_{S}}(t), \\
& \frac{d \rho_{I, k}(t)}{d t}=\beta \rho_{S, k}(t) \rho_{I, k}(t)-\mu \rho_{I, k}(t)-d \rho_{I, k}(t)-p \rho_{I, k}(t)+p \frac{k}{\langle k\rangle} \bar{\rho}_{I}(t), \\
& \frac{d \rho_{R, k}(t)}{d t}=\mu \rho_{I, k}(t)-d \rho_{R, k}(t)-p \rho_{R, k}(t)+p \frac{k}{\langle k\rangle} \overline{\rho_{R}}(t), \\
& \frac{d \rho_{k}(t)}{d t}=\eta\left(1-\frac{\rho_{k}(t)}{\theta_{k}}\right) \rho_{k}(t)-p \rho_{k}(t)+p \frac{k}{\langle k\rangle} \bar{\rho}(t)
\end{aligned}
$$

where $\overline{\rho_{A}}(t)=\sum_{k} P(k) \rho_{A, k}(t)(A=S, I$ or $R)$ denotes the average number of susceptible (infected or recovered) individuals at time $t$, and $\bar{\rho}(t)=\overline{\rho_{S}}(t)+\overline{\rho_{I}}(t)+\overline{\rho_{R}}(t)$ is the average population size of each patch. 
Setting the left side of Eq. (5d) equal to zero, the stationary state equation of the population demographics is

$$
\eta\left(1-\frac{\rho_{k}}{\Theta_{k}}\right) \rho_{k}-p \rho_{k}+p \frac{k}{\langle k\rangle} \bar{\rho}=0 .
$$

By calculating the solution of Eq. (6), we get

$$
\rho_{k}=\frac{(\eta-p)+\sqrt{(p-\eta)^{2}+4 \eta p \mathrm{H}_{k} / \Theta_{k}}}{2 \eta / \Theta_{k}}
$$

where $\mathrm{H}_{k}=\frac{k}{\langle k\rangle} \bar{\rho}\left(\mathrm{H}_{k}\right.$ is actually a solution of $\rho_{k}$ in the case of just considering homogenous random migration without demographics [7, 8]). From Eq. (7), we know that the final population distribution is not only dependent on network structure (see the definitions of $\mathrm{H}_{k}$ and $\Theta_{k}$ ), but also on dynamics parameters $\eta$ and $p$. For the special case of $\eta=p, \rho_{k}$ can be reduced to

$$
\rho_{k}=\left(\Theta_{k} \mathrm{H}_{k}\right)^{1 / 2}=\left(\frac{k^{(\alpha+1) \bar{\Theta} \bar{\rho}}}{\left\langle k^{\alpha}\right\rangle\langle k\rangle}\right)^{1 / 2} .
$$

This equation explicitly indicates how and to what degree the capacity and migration influence the population in each patch in the stationary limit. Furthermore, by multiplying Eq. (8) by $P(k)$ and summing over $k$, and then after some simple algebra, we obtain the following equation

$$
\bar{\rho}=\frac{\left\langle k^{(\alpha+1) / 2}\right\rangle^{2}}{\left\langle k^{\alpha}\right\rangle\langle k\rangle} \bar{\Theta} .
$$

At this point, inserting Eq. (9) into Eq. (8), $\rho_{k}$ can also be written as

$$
\rho_{k}=\frac{\left\langle k^{(\alpha+1) / 2}\right\rangle k^{(\alpha+1) / 2}}{\left\langle k^{\alpha}\right\rangle\langle k\rangle} \bar{\Theta} .
$$

Eq. (9) indicates the relation between the average population in each patch and the initial average capacity over the system in the stationary limit. In other words, for any given initial population capacity, we can obtain the final population size of the system. Eq. (10) expresses the population distribution over different patch degrees in the stationary limit. These results will be used in the following epidemic threshold analysis and dynamics behaviours simulations. In order to understand intuitively these relations, the curve of Eq. (9) is plotted in Fig. $2(\bar{\rho} / \bar{\Theta}$ is a function of $\alpha)$, and the curves of $\rho_{k}$ are plotted according to Eq. (10) in Fig. 3.

We can obtain some interesting results from Fig.2 that: (i) when $\alpha=1, \bar{\rho}$ reaches the maximum $\bar{\rho}=\bar{\Theta}$, meaning the population size of the system in the stationary limit equal to the initial capacity of system regardless of growth, migration rate, and network structure; (ii) for any given value of $\alpha(0<\alpha<1)$, there exists a corresponding value of $\alpha(1<\alpha<2)$ 
rendering two equal values of $\bar{\rho}$. But at the same time, here it is necessary to stress that these results are derived only in the case of $\eta=p$.

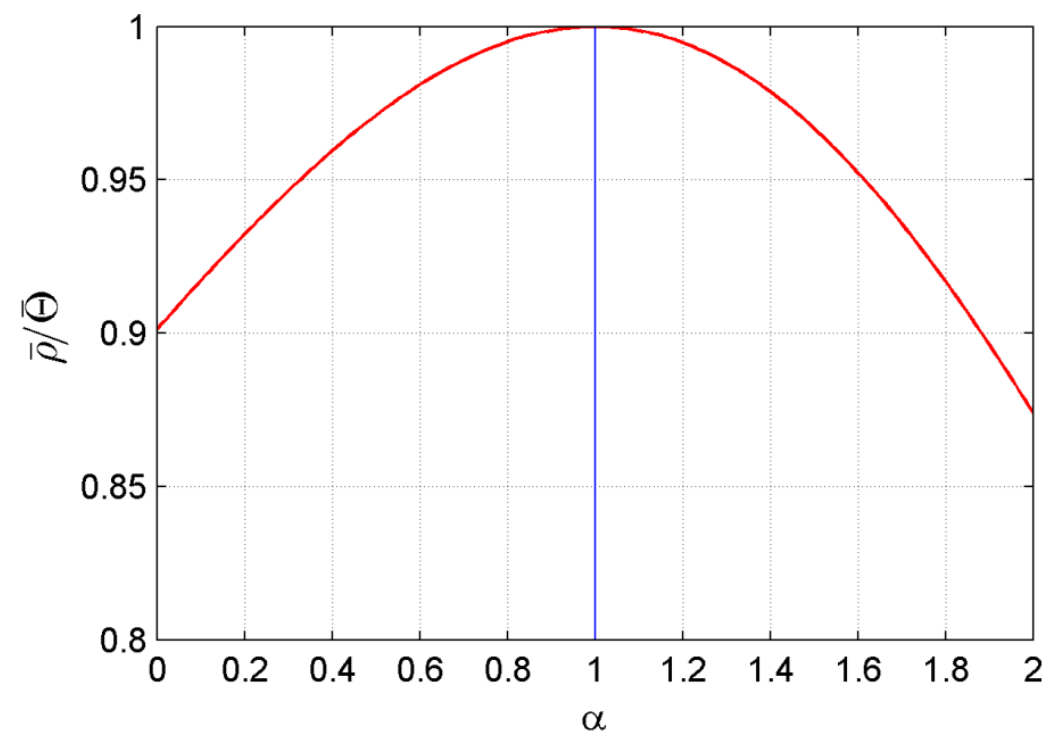

Fig. 2 The ratio $\bar{\rho} / \bar{\Theta}$ as a function of $\alpha$ (plotted according to Eq. (9)). The underlying network generated by the uncorrelated configuration model [30] has $V=1000$ nodes with power-law degree distribution $P(k) \sim k^{-2.5}\left(k_{\min } \leq k \leq k_{\max }\right)$ with $k_{\min }=3$ and $k_{\max }=57$, and the average degree $\langle k\rangle=5.8$.

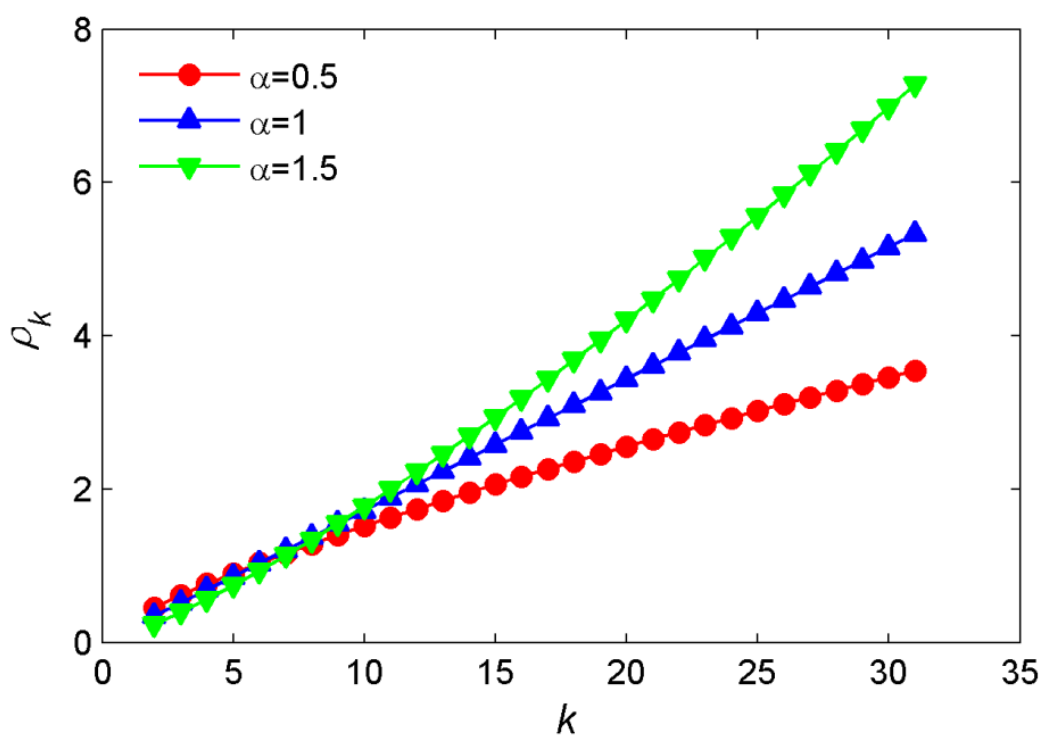

Fig. 3 The final population size of each patch $\rho_{k}$ as a function of its degree $k$ (plotted according to Eq. (10) with $\bar{\Theta}=1$ ). The underlying network is the same as that in Fig. 2.

The relation between the final population size of each patch and its degree $k, \rho_{k}$, depends greatly on the heterogeneity of capacity. A smaller heterogeneity (e.g., $\alpha=0.5$ ) leads to little difference in the population size among patches with different degree $k$, and a larger one (e.g., $\alpha=1.5$ ) enlarges such difference (see Fig. 3). The special case of $\alpha=1$ implies an exactly linear relation between $\rho_{k}$ and $k$. 
To clear and easy to find for readers, all variables used in the metapopulation model are listed in Table 2.

Table 2 Metapopulation model variables

\begin{tabular}{|c|c|c|}
\hline Variable & Meaning & $\begin{array}{l}\text { Value used in } \\
\text { simulation }\end{array}$ \\
\hline$k$ & degree of a node (patch) & {$[3 ; \sqrt{V}]$} \\
\hline$\langle k\rangle$ & $\begin{array}{l}\text { average degree of a metapopulation } \\
\text { network }\end{array}$ & $\langle k\rangle=5.8$ \\
\hline V & total number of nodes & $V=10^{3}$ \\
\hline$P(k) \sim k^{-\gamma}$ & $\begin{array}{l}\text { node degree distribution of } \quad \text { a } \\
\text { metapopulation network }\end{array}$ & $\gamma=2.5$ \\
\hline $\bar{\Theta} ; \Theta_{i}$ & $\begin{array}{l}\text { average capacity of a node; capacity of } \\
\text { node } i\end{array}$ & $\bar{\Theta}=100$ \\
\hline$\Theta_{k}=\frac{k^{\alpha}}{\left\langle k^{\alpha}\right\rangle} \bar{\Theta}$ & $\begin{array}{l}\text { capacity of a node with degree } k \\
\text { heterogeneous level exponent of the } \\
\text { capacity of nodes }\end{array}$ & {$\left[\begin{array}{ll}0 & 2\end{array}\right]$} \\
\hline $\begin{array}{l}\rho_{S, i}(t) ; \rho_{I, i}(t) ; \rho_{R, i}(t) \\
\rho_{i}(t)\end{array}$ & $\begin{array}{l}\text { number of susceptible, infected and } \\
\text { recovered individuals in node } i \text { at time } t \\
\text { total number of individuals in node } i \text { at } \\
\text { time } t\end{array}$ & $\rho_{i}(0)=0.8 \times \Theta_{i}$ \\
\hline $\begin{array}{l}\rho_{S, k}(t) ; \rho_{I, k}(t) ; \rho_{R, k}(t) \\
\rho_{k}(t)\end{array}$ & $\begin{array}{l}\text { average number of susceptible, infected } \\
\text { and recovered individuals in nodes with } \\
\text { degree } k \text { at time } t \\
\text { average population size of each node with } \\
\text { degree } k \text { at time } t\end{array}$ & $\rho_{k}(0)=0.8 \times \Theta_{k}$ \\
\hline $\begin{array}{l}\bar{\rho} \\
\rho_{k}\end{array}$ & $\begin{array}{l}\text { average final population size of nodes } \\
\text { average final population size of nodes with } \\
\text { degree } k\end{array}$ & \\
\hline
\end{tabular}

\section{THE DISEASE-FREE EQUILIBRIUM AND THRESHOLD}

In order to easily obtain the threshold of the system, we consider the reduced system of system (5)

$$
\begin{aligned}
& \frac{d \rho_{I, k}(t)}{d t}=\beta \rho_{S, k}(t) \rho_{I, k}(t)-\mu \rho_{I, k}(t)-d \rho_{I, k}(t)-p \rho_{I, k}(t)+p \frac{k}{\langle k\rangle} \bar{\rho}_{I}(t), \\
& \frac{d \rho_{R, k}(t)}{d t}=\mu \rho_{I, k}(t)-d \rho_{R, k}(t)-p \rho_{R, k}(t)+p \frac{k}{\langle k\rangle} \overline{\rho_{R}}(t), \\
& \frac{d \rho_{k}(t)}{d t}=\eta\left(1-\frac{\rho_{k}(t)}{\Theta_{k}}\right) \rho_{k}(t)-p \rho_{k}(t)+p \frac{k}{\langle k\rangle} \bar{\rho}(t) .
\end{aligned}
$$

The dynamics of $\rho_{S, k}(t)$ can be simply obtained from equation $\rho_{S, k}(t)=\rho_{k}(t)-$ $\rho_{I, k}(t)-\rho_{R, k}(t)$. By analysing the stability of Eq. (11) at the disease-free equilibrium point, the condition for an epidemic outbreak (i.e., spreading threshold) can be obtained. Let the right sides of Eq. (11) be equal to zero, and taking $\rho_{I, k}=\rho_{R, k}=0$, the disease-free equilibrium is then given by 


$$
\rho_{I, k}^{*}=0, \rho_{R, k}^{*}=0, \rho_{S, k}^{*}=\rho_{k}^{*}=\rho_{k} .
$$

Linearizing Eq. (11) around the disease-free equilibrium (Eq. (12)), the Jacobian matrix of the system is a block matrix given by

$$
J=\left[\begin{array}{ccc}
J_{1} & 0 & 0 \\
J_{2} & J_{3} & 0 \\
0 & 0 & J_{4}
\end{array}\right]
$$

where each block is a $m \times m$ matrix with $m$ being the number of degrees in the metapopulation, 0 is the null matrix, and

$$
\begin{aligned}
& J_{1}=\left(\beta \rho_{S, k}^{*}-\mu-d-p\right) E+p C, \\
& J_{2}=\mu E, \\
& J_{3}=-(d+p) E+p C, \text { and } \\
& J_{4}=\left(-\frac{2 \eta \rho_{k}^{*}}{\Theta_{k}}+\eta-p\right)+p C
\end{aligned}
$$

where $E$ is the identity matrix and $C$ is the connectivity matrix [18-19] given by

$$
C_{k k^{\prime}}=\frac{k P\left(k^{\prime}\right)}{\langle k\rangle} \text {. }
$$

Obviously, $C$ is a rank-one matrix and has an eigenvalue $\lambda=0$ with algebraic multiplicity $m-1$, and an eigenvalue $\lambda=1$. The lower triangular structure of $J$ implies that the spectrum of $J$ is the union of the three blocks of $J_{1}, J_{3}$ and $J_{4}$. Its characteristic polynomial can be represented as $f(\lambda)=f_{1}(\lambda) f_{3}(\lambda) f_{4}(\lambda)$ with $f_{1}(\lambda), f_{3}(\lambda)$ and $f_{4}(\lambda)$ being the characteristic polynomials of block $J_{1}, J_{3}$ and $J_{4}$ respectively.

From the knowledge of the eigenvalues of $C$, it can be derived that $f_{3}(\lambda)=(\lambda+$ $d)(\lambda+d+p)^{m-1}$, so the largest eigenvalue of $J_{3}, \lambda_{\max }^{3}=-d<0$. Analysing the matrixes $J_{1}$ and $J_{4}$ by using a general interlacing theorem of eigenvalues for perturbations of a diagonal matrix by rank-one matrices [31], $f_{1}(\lambda)$ and $f_{4}(\lambda)$ have respectively $m$ simple real roots and the largest root of $f_{1}(\lambda)$ (i.e., the largest eigenvalue of matrix $J_{1}$ ) satisfies $\lambda_{\max }^{1}>\beta \rho_{k_{\max }}-$ $(\mu+d+p)$, and the largest root of $f_{4}(\lambda)$ (i.e., the largest eigenvalue of matrix $\left.J_{4}\right)$ satisfies $\lambda_{\max }^{4}>\eta-p-2 \eta \frac{\left\langle k^{(\alpha+1) / 2}\right\rangle}{\langle k\rangle}\left(k_{\max }\right)^{(1-\alpha) / 2} \quad$ if $\quad \alpha>1 \quad$ and $\lambda_{\max }^{4}>\eta-p-2 \eta \frac{\left\langle k^{(\alpha+1) / 2}\right\rangle}{\langle k\rangle}\left(k_{\min }\right)^{(1-\alpha) / 2}$ if $\alpha<1$. Notice that the spectral property of matrix $J_{4}$ has no relation with the invasion threshold and just determines the population dynamics. It does not make sense if the population becomes divergent, so we control $\lambda_{\max }^{4}<$ 0 here. It is difficult to derive theoretical expression of $\lambda_{\max }^{4}$, and hence we test on each network that our simulations ensure that $\lambda_{\max }^{4}<0$ by selecting carefully the values of 
parameters. In fact, if we let $\eta=p$, the condition $\lambda_{\max }^{4}<0$ will always hold in a limited scale-free network, because the final population size is bounded according to Eq. (9). An example is shown in Fig. 4.

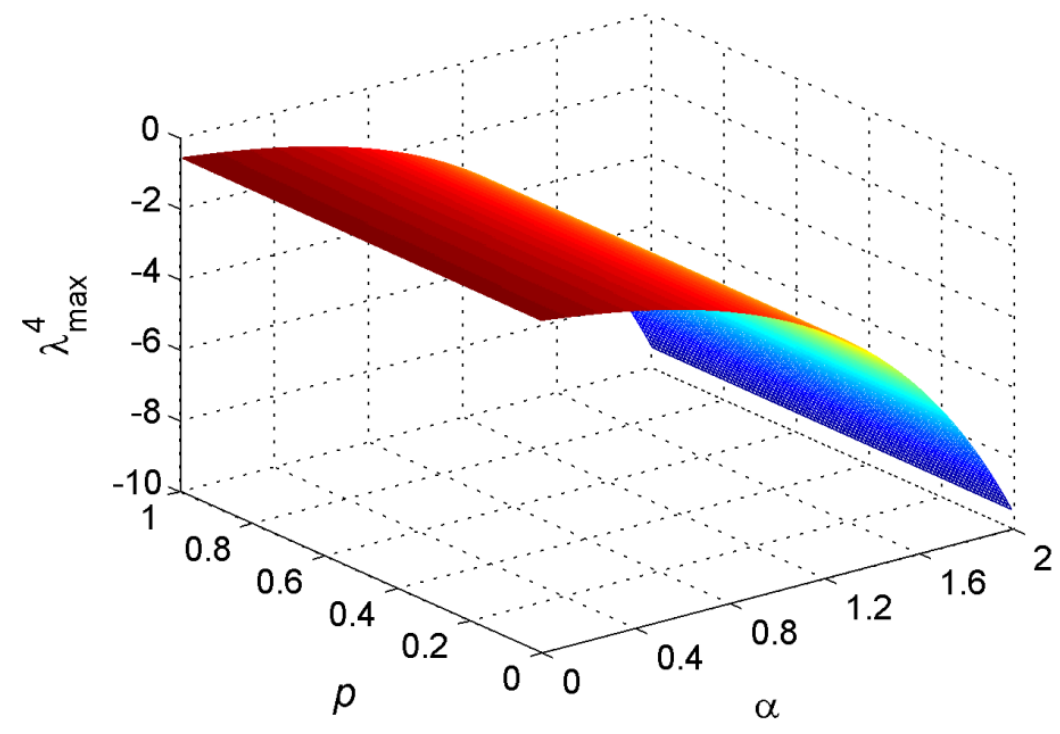

Fig. 4 The maximum eigenvalue of $J_{4}, \lambda_{\max }^{4}$, as a function of $\alpha$ and $p$ in the case of $\eta=p$. The underlying network is the same network as that in Fig. 2.

Based on the preceding analysis, a sufficient condition for the disease-free equilibrium to be unstable and reach to an endemic state finally is given by

$$
\beta \rho_{k_{\max }}-(\mu+d+p)>0 .
$$

By inserting Eq. (7) into Eq. (14) and replace $\Theta_{k}$ and $\mathrm{H}_{k}$ in the result equation with their respective expression, we obtain the general condition for epidemic outbreak as

$$
\frac{\left(k_{\max }\right)^{\alpha} \bar{\Theta}}{2 \eta\left\langle k^{\alpha}\right\rangle}\left[(\eta-p)+\sqrt{(p-\eta)^{2}+4 \eta p \frac{\left\langle k^{\alpha}\right\rangle\left(k_{\max }\right)^{(1-\alpha)}}{\langle k\rangle} \overline{\bar{\rho}}}\right]>\frac{(\mu+d+p)}{\beta} .
$$

This inequality implies an implicit expression for the threshold with respect to the final average population density $\bar{\rho}$ and average capacity $\bar{\Theta}$. Unfortunately, $\bar{\rho}$ cannot be given directly in advance because the population size of the system is time-varying. When using this threshold condition, we need first to calculate the value of $\rho_{k}$ according to Eq. (7) based on the given initial values of the system, and further derive $\bar{\rho}$ by taking the average of $\rho_{k}$. Thus it is impossible to use this threshold condition directly even if the initial parameters are given.

If we consider the special case of $\eta=p$, combing Eq. (10) and Eq. (14), it is easy to obtain an explicit expression for the epidemic threshold with respect to $\bar{\Theta}_{c}$

$$
\bar{\Theta}_{\mathrm{c}} \geq \frac{\left\langle k^{\alpha}\right\rangle\langle k\rangle}{\left(k_{\max }\right)^{(\alpha+1) / 2}\left\langle k^{(\alpha+1) / 2}\right\rangle} \frac{(\mu+d+p)}{\beta} .
$$


Comparing with Eq. (15), this threshold expression is very simple, and more importantly we can use this condition directly by giving the initial values of system. Clearly, for a given metapopulation network, and fixed dynamics parameters $\mu, d, p$, and $\beta$, as well as exponent $\alpha$, a high enough initial average capacity per patch $\bar{\Theta}$ guarantee the instability of the disease-free equilibrium. This also shows that the level of heterogeneity of capacity governed by the exponent $\alpha$, and migration rate $p$, have significant influence on the threshold.

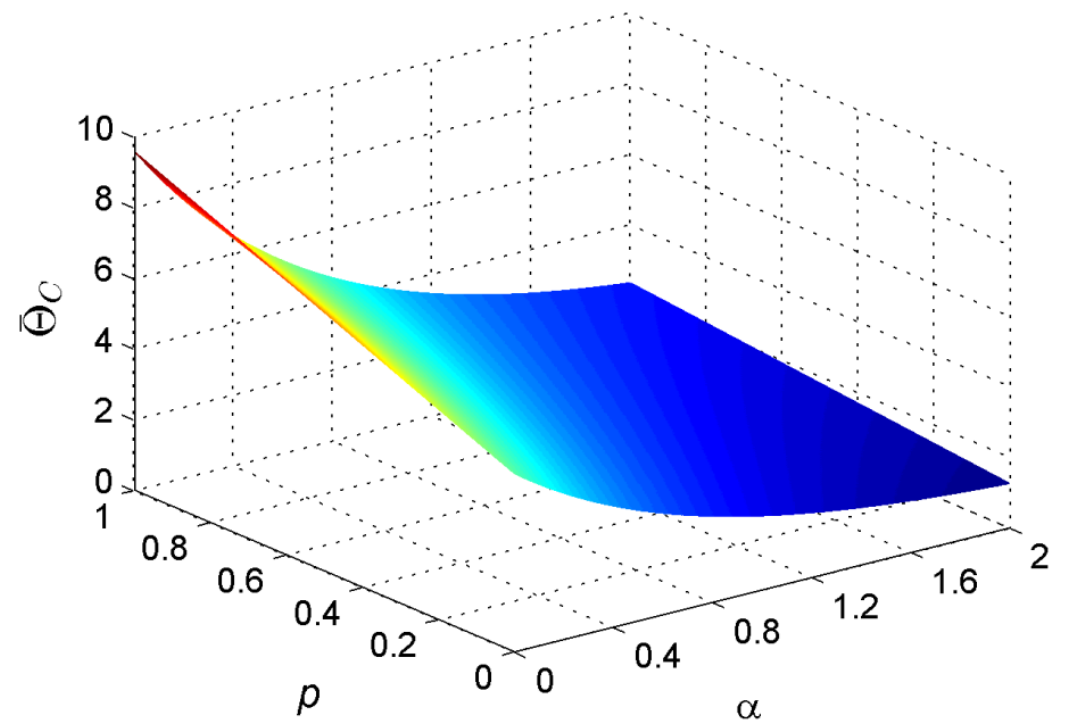

Fig. 5 The threshold $\bar{\Theta}_{c}$ as a function of $\alpha$ and $p$. The dynamics parameters are $d=0.1, \beta=0.1$ and $\mu=1$, and the underlying network is the same network as that in Fig. 2.

To show this clearly, we plot the dependence of threshold $\bar{\Theta}_{\mathrm{c}}$ on parameters $\alpha$ and $p$ according to Eq. (16). $\bar{\Theta}_{\mathrm{c}}$ is a decreasing function of $\alpha$ and an increasing function of $p$, which means the high heterogeneity of capacity favours an epidemic outbreak and high migration level acts against it in terms of epidemic threshold. Moreover, the heterogeneity of capacity has a greater impact on the threshold than migration rate (see Fig. 5).

We can also understand this invasion condition from the perspective of the basic reproduction number $R_{0}$ (i.e., the average number of secondary infections produced generated by one primary infected individual in a fully susceptible [32]). Clearly, if $R_{0}>1$, an epidemic can spread in the population. Rewriting Eq. (16) as $\frac{\beta \bar{\theta}}{(\mu+d+p)} \frac{\left(k_{\max }\right)^{(\alpha+1) / 2}\left\langle k^{(\alpha+1) / 2}\right\rangle}{\left\langle k^{\alpha}\right\rangle\langle k\rangle} \geq 1$, we will get $R_{0}$ as following

$$
R_{0}=\frac{\beta \bar{\Theta}}{(\mu+d+p)} \frac{\left(k_{\max }\right)^{(\alpha+1) / 2}\left\langle k^{(\alpha+1) / 2}\right\rangle}{\left\langle k^{\alpha}\right\rangle\langle k\rangle} .
$$

This in fact is the basic reproduction number in a patch of $k_{\max }$ degree with recovered rate $\mu$, death rate $d$, and migration rate $p$ to patches of different degrees. But here it is necessary to emphasize that this expression does not take into account those infected individuals coming 
from patches with the same degree $k_{\max }$, which means that the actual value of $R_{0}$ in patches with degree $k_{\max }$ may be somewhat greater than the value given by Eq. (17). Equivalently, the actual threshold of $\bar{\Theta}_{\mathrm{c}}$ in patches with degree $k_{\max }$ may be somewhat less than the threshold given by Eq. (16). So condition Eq. (16) guarantees the spread of an epidemic in those patches with the maximum degree and allows the disease to eventually reach all patches [18].

\section{SIMULATION RESULTS AND ANALYSIS}

In order to validate the theoretical predictions on the epidemic conditions in Section 3 and to further study the impact of demographics and migration on epidemic behaviours above the threshold, we perform an extensive set of Monte Carlo simulations on an artificial network. Each simulation starts with a randomly selecting patch as infected source and results are based on an average of 50 independent simulation runs.

The artificial network has $V=1000$ nodes symbolizing a system of 1000 inter-connected patches, and it is generated by the uncorrelated configuration model [30]. The degree distribution of this network follows power-law function $P(k) \propto k^{-2.5}$ and average degree is $\langle k\rangle=5$.8. In each discrete time step, the rates $b, d, \eta, p, \beta$, and $\mu$ have to be respectively replaced by probabilities $b \tau, d \tau, \eta \tau, p \tau, \beta \tau$, and $\mu \tau$, where $\tau$ is the small enough unitary time scale. In our simulations, we set demographic rates $d=0.1, b=\eta+d$ and $\eta=p$, and infection dynamics rates $\beta=0.1$ and $\mu=1$, the unitary time scale $\tau=0.01$ if not otherwise specified.

It is important to note that if the heterogeneity level of capacity is extremely low or high, some nodes in the system perhaps have too overtaking population burden because of their limit capacity, which represents $\left(b-\frac{\eta \rho_{k}(t)}{\theta_{k}}\right)<0$ (a negative real birth rate) in Eq. (5). In simulation process, we consider this case as a capacity limited death rate due to competition for resource.

Fig. 6 shows the simulation thresholds as a function for heterogeneity exponent of capacity $\alpha$ with the theoretical values given by Eq. (16) for different migration rates $p$ ((a) $p=0.2$ and (b) $p=0.5$ ). Firstly, the simulated values approximately match the theoretical ones, and at the same time, with the increase of heterogeneous exponent $\alpha$, threshold $\bar{\Theta}_{c}$ decreases gradually (see Fig. 6 (a) and (b) respectively), and instead that increases with increasing migration rate (e.g., from $p=0.2$ to $p=0.5$ ). This means that the heterogeneity of capacity benefits epidemic spreading and migration does not in terms of epidemic threshold. This validates the correctness of the theoretical findings on invasion condition. Secondly, the 
simulated circles are located sometimes below the curve of theoretical threshold which verifies the analysis results that actual threshold may somewhat lower than theoretical threshold given by Eq. (16).
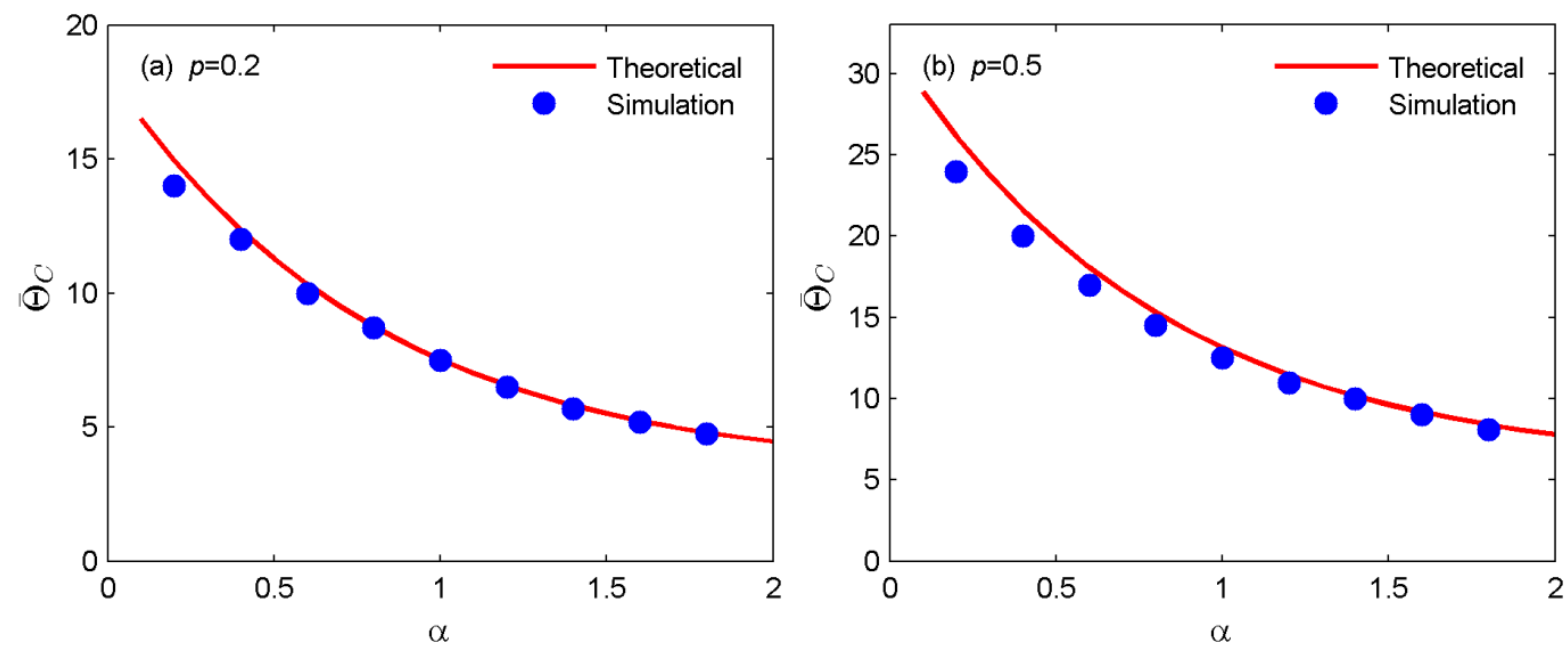

Fig. 6 Epidemic threshold $\bar{\Theta}_{\mathrm{c}}$ as a function of heterogeneity exponent $\alpha$ with $\beta=0.01$ and $\mu=0.1$. Blue circles and red line represent the simulated and theoretical values of the threshold respectively. The underlying network is the same network as that in Fig. 2, and the theoretical values of the threshold are given by Eq. (16).

Monte Carlo simulations also allow for the study of the spreading behaviours above the epidemic threshold. In the following, we further perform some Monte Carlo simulations in the same network to investigate the impact of the heterogeneity of capacity and migration rate on epidemic behaviours above epidemic threshold. In each time step, we monitor the normalized average infection denoted as $\bar{\rho}_{I}(t) / \bar{\rho}(t)$ which mainly reflects the temporal behaviours of infection prevalence, and the average of population $\bar{\rho}(t)$ which indicates the evolution process of the population. We only compare simulation results between different cases that have the same population in the stationary limit. The initial values are: the average capacity $\bar{\Theta}=100$ (such that the maximum number of individuals in the system equals $\bar{\Theta} \times V=10^{5}$ ), the initial $\bar{\rho}(0)=0.8 \times \bar{\Theta}$. When compared of the prevalence at different levels of heterogeneity of capacity, we carefully choose three pairs values of $\alpha$ (Case (a) Case (c)) to render the system having almost the same theoretical value of $\bar{\rho}$ according to Eq. (9) for each case (See Table 3). To better illustrate the different impacts clearly for each case, we also track the difference quantity of infection

$$
\Delta\left(\frac{\overline{\rho_{I}}(t)}{\bar{\rho}(t)}\right)=\left.\frac{\overline{\rho_{I}}(t)}{\bar{\rho}(t)}\right|_{\alpha 1}-\left.\frac{\overline{\rho_{I}}(t)}{\bar{\rho}(t)}\right|_{\alpha 2},
$$

where $\alpha 1$ and $\alpha 2$ are respectively the larger and the smaller value of $\alpha$ in each case. 
The simulated curves of $\overline{\rho_{I}}(t) / \bar{\rho}(t)$ for each case are shown in Fig. $7\left(\Delta\left(\overline{\rho_{I}}(t) / \bar{\rho}(t)\right)\right.$ in the inset), and the simulated values of $\bar{\rho} \times V$ are demonstrated in Table 3 .

It can be clearly seen from Fig. 7 that the heterogeneity of capacity mainly affects the epidemic speed. That is to say the heterogeneity of capacity enhances epidemic spreading speed at early time steps and such impact is more pronounced when differentiation of the heterogeneity is higher (comparing Fig. 7 (a) Fig. 7 (c) and the inset of each). On the other hand, the heterogeneity has almost no influence on the infection size in the stationary limit. This phenomenon can be explained as follows: at early time steps, epidemic spreading only occurs in a small fraction of patches with larger degree. One reason is infection cannot reach to most patches due to short evolution time, and another is that patches with larger degree are easier and earlier to be affected by infection because of random migration and their greater connectivity. Furthermore, the heterogeneity of capacity guarantees these patches with larger degree have more individuals which benefits epidemic spreading. So epidemic spreads quickly in the metapopulation with high heterogeneous capacity. But as time goes on, most patches are seeded by infection such that epidemic prevalence mainly depends on the infectivity of the disease and recover rate of individuals, which results in the same finial infection size.

Further from Table 3, we can see that in each case both two simulated values of $\bar{\rho} \times V$ are approximately equal to the theoretical value. Case (c) as an example, the theoretical value of $\bar{\rho} \times V$ is $9.84 \times 10^{4}$, and the simulated one is $9.76 \times 10^{4}$ for $\alpha=0.63$ and $9.70 \times 10^{4}$ for $\alpha=1.35$. This approximately validates the theoretical finding of the final population size given by Eq. (9) although there are somewhat differences between theoretical and simulated values.

Table 3. The theoretical and simulated values of the final population size $\bar{\rho} \times V$ for Cases (a) (c). Theoretical values of $\bar{\rho} \times V$ are given by Eq. (9)

\begin{tabular}{c|c|c|c}
\hline \hline \multirow{2}{*}{ Case } & Value of $\alpha$ & \multirow{2}{*}{ Theoretical $\bar{\rho} \times V$} & Simulation $\bar{\rho} \times V$ \\
\hline \multirow{2}{*}{ (a) } & $\alpha=0.10$ & \multirow{2}{*}{$9.17 \times 10^{4}$} & $9.07 \times 10^{4}$ \\
\cline { 2 - 2 } (b) & $\alpha=1.80$ & & $9.12 \times 10^{4}$ \\
\cline { 2 - 2 } (c) & $\alpha=0.38$ & \multirow{2}{*}{$9.57 \times 10^{4}$} & $9.46 \times 10^{4}$ \\
\cline { 2 - 2 } & $\alpha=0.63$ & \multirow{2}{*}{$9.84 \times 10^{4}$} & $9.47 \times 10^{4}$ \\
\cline { 2 - 2 } & $\alpha=1.35$ & & $9.76 \times 10^{4}$ \\
\hline \hline
\end{tabular}



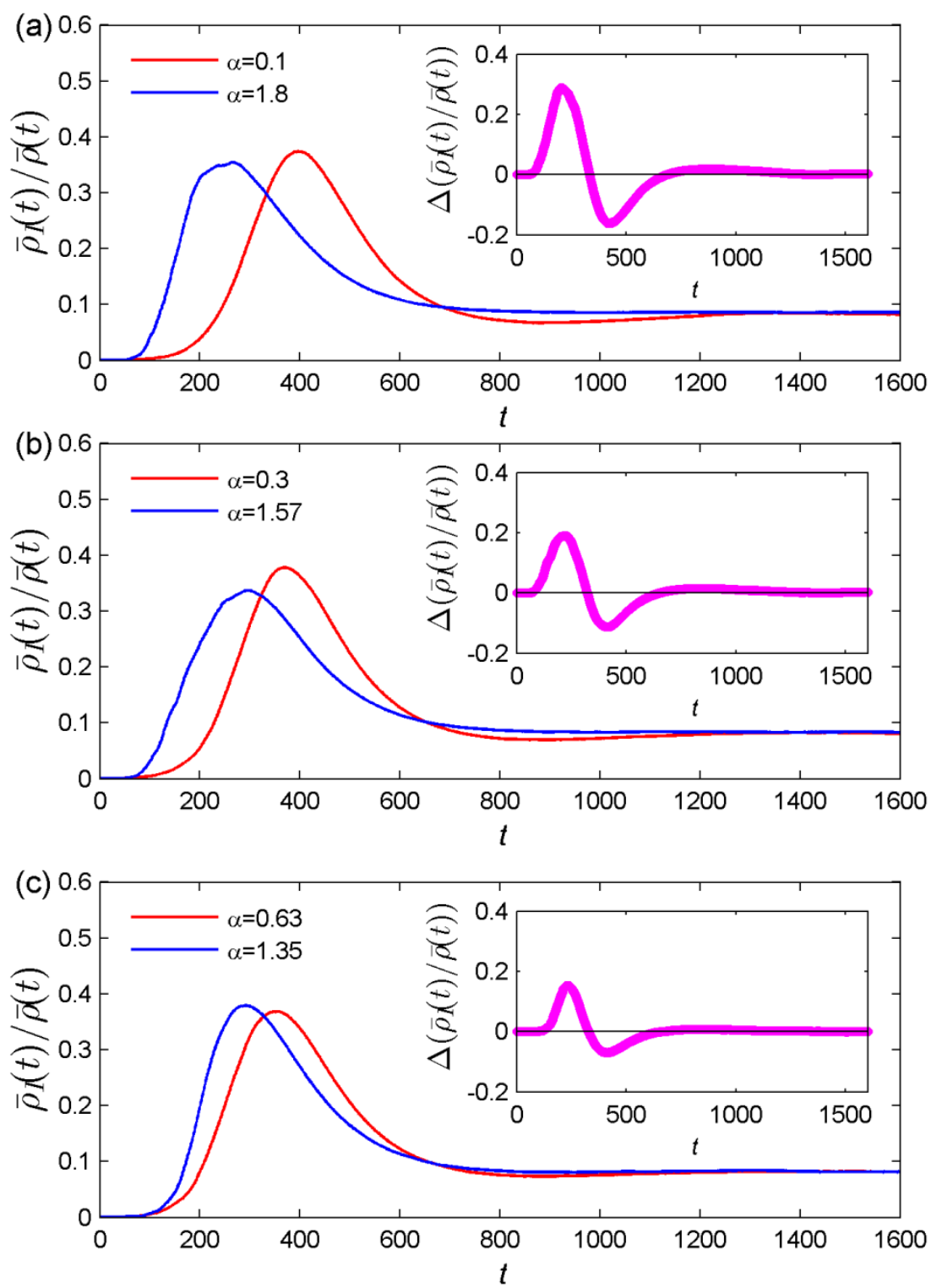

Fig. 7 Time evolutions of the normalized average infection density $\bar{\rho}_{I}(t) / \bar{\rho}(t)$ of two distinct values of $\alpha$ and the difference of them $\Delta\left(\bar{\rho}_{I}(t) / \bar{\rho}(t)\right)$ (the inset) for Case (a) Case (c) in the same network as that in Fig. 2.

In order to further explore how the migration rate affects the temporal epidemic prevalence, we perform another set of simulations on the same network with different migration rates (i.e., $p=0.1,0.5$, and 0.8 ) for several scenarios with $\alpha=0.5,1,1.5$. Obviously, for each scenario, the system has the same final population size according to Eq. (9) although it has different migration rates.

The simulations show that in the initial evolution stage, the larger the migration rate $p$ is, the higher the infection density is, which can be explained by the larger migration rate making the infected individuals move between patches faster. In the middle stage, with time going on, the infected individuals will reach the most patches for all the cases of migration rate $p$, at this 
point, infection size is larger in the case of smaller $p$ and is smaller in the case of larger $p$ (see Fig. 8). The reason is that for the cases of $p$ that early reach the peak of outbreak (e.g., $p=0.8$ ), infection density begins to decrease due to the recovering process of the infected individuals. In the later stage, epidemic spreading reaches an endemic state, we find an interesting result: greater values of $p$ lead to a larger final infection size for lower and higher heterogeneities level of capacity (see the inset of Fig. 8 (a) and (c)) and the final infection sizes are almost the same for the middle heterogeneity level (see the inset of Fig. 8 (b)).
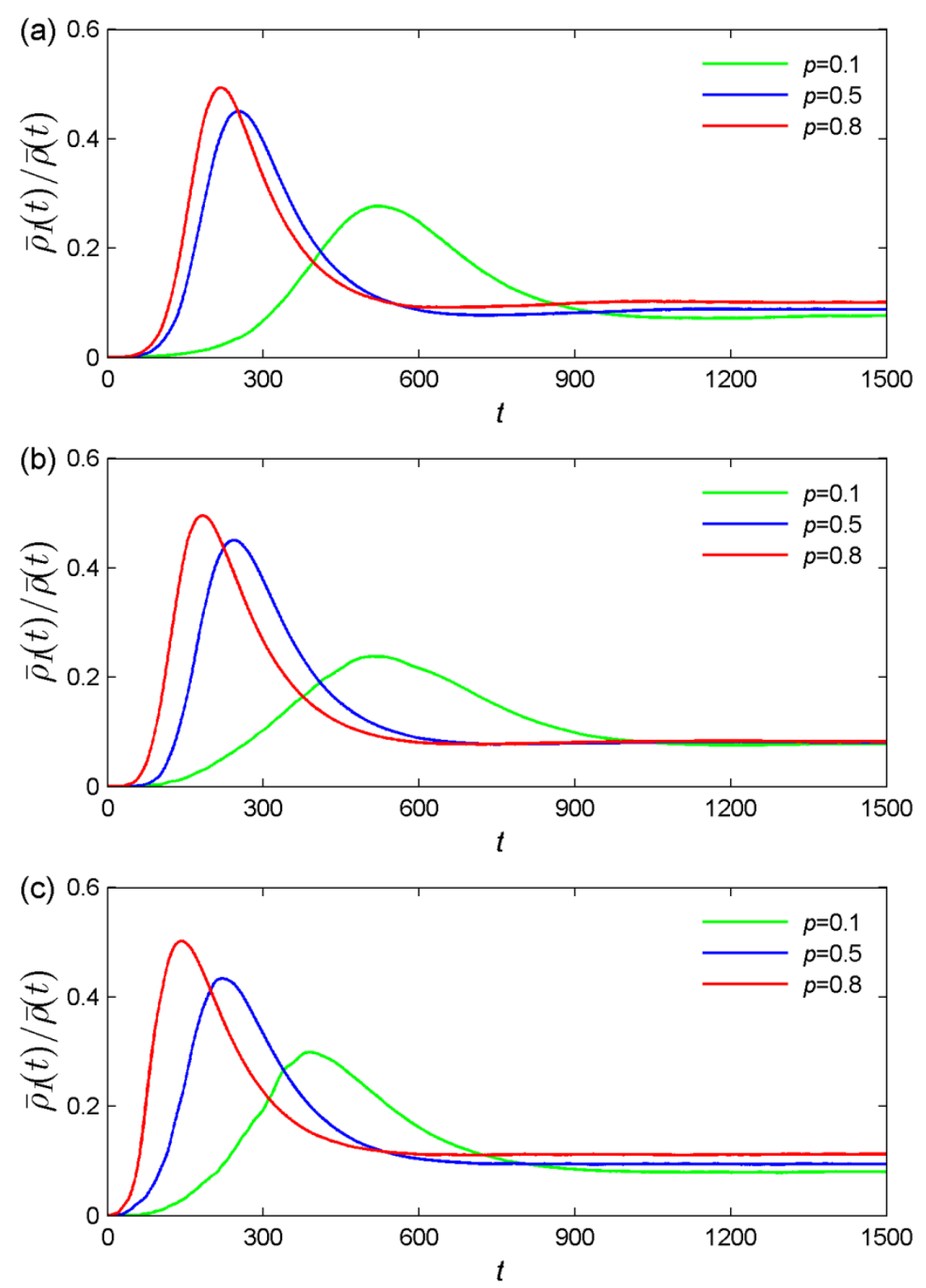

Fig. 8 Time evolutions of the normalized average infection density $\bar{\rho}_{I}(t) / \bar{\rho}(t)$ with different migration rates $p=0.1,0.5,0.8$ for several scenarios: (a) $\alpha=0.5$, (b) $\alpha=1$, (c) $\alpha=1.5$ in the same network as that in Fig. 2. 
To further test the correctness and robustness of this surprising finding at more general heterogeneity level of capacity, we simulate the final infection size at a larger range of $\alpha$ (i.e., $0 \leq \alpha \leq 2$ ) and the results are shown in Fig. 9.

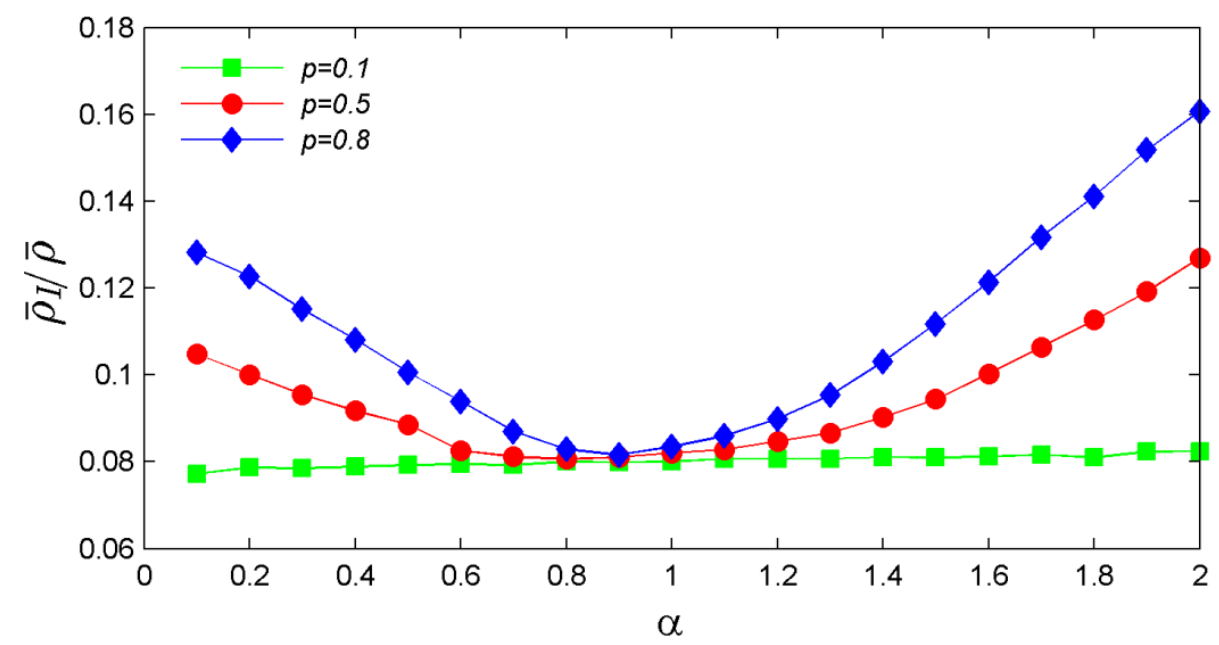

Fig. 9 The normalized average infection density in the stationary limit as a function of $\alpha$ with different migration rate $p=0.1,0.5,0.8$ in the same network as that in Fig. 2.

Infection density in the stationary limit influenced by migration rate depending on the heterogeneity level of capacity is shown in Fig. 9. Our simulation results show that for the low (e.g., $0<\alpha<0.8$ ) and high (e.g., $1.1<\alpha<2$ ) heterogeneity levels of capacity, the greater the value of $p$ is, the larger final infection size, and with the heterogeneity level tending to two extremes, this result becomes more pronounced. However, when the heterogeneity level is intermediate (e.g., $0.8 \leq \alpha \leq 1.1$ ), the final sizes are almost the same for different $p$ values. These results also further illustrate the phenomena observed in Fig. 8. The reason may be as follows: for low heterogeneity levels $(0<\alpha<0.8)$, the capacity of each node tends to be homogeneous, the large random migration rate will make individuals move to nodes with higher degree faster than the case of small migration rate, which causes these nodes to have a heavier burden on the population and result in a higher capacity limited death rate. In this case, more new susceptible individuals continuously enter the system through lower degree nodes, which leads to a higher infection density. When the heterogeneity levels are higher $(1.1<\alpha<2)$, the capacity of each node is polarized. Thus the birth of new susceptible individuals mainly occurs in higher degree nodes with larger capacity, the larger random migration rate results in an overtaking population in the low degree nodes and further causes a higher capacity limited death rate in these nodes. In this case, more new susceptible individuals will go in the system through the higher degree nodes. But for an intermediate level $(0.8 \leq \alpha \leq 1.1)$, the capacity of each node varies almost 
linearly with node degree, which means each node nearly has the same real birth and death rates and no capacity limited death. So the new susceptible individuals entering the system are almost the same regardless of the migration rate, which leads to the almost same final infection density.

\section{CONCLUSIONS AND DISCUSSIONS}

We have presented a theoretical framework to study epidemic spreading in a heterogeneous spatial metapopulation with migration and demographics. In this framework, a modified SIR epidemic model was formulated based on mean-field approach by incorporating random migration and logistic population dynamics in each patch with a heterogeneous capacity. A theoretical invasion threshold was derived and it is shown that the heterogeneity of capacity of population drastically decreases the threshold, and instead the increase of the migration rate slightly increases the threshold. This theoretical result was tested with a set of Monte Carlo simulations on a UCM network. Furthermore, we also have studied by Monte Carlo simulations how the heterogeneity of capacity and migration rate impact the epidemic behaviours above epidemic threshold under the condition that system has the same stationary population size. It is shown that both the heterogeneity of capacity and migration benefit epidemic spreading at early stage. But for the final infection size, the former factor has no impact on it, and the impact of the later factor depends on the heterogeneity level of capacity.

Because of the complexity of our epidemic model, it is very difficult to thoroughly analyze the model by theoretical means. So we theoretically derived the epidemic threshold in terms of the average capacity of population, and studied the epidemic behaviours above the epidemic by Monte Carlo simulations. Moreover, in order to compare more fairly the impact of distinct heterogeneity level of capacity and migration on epidemic prevalence, we carefully select values of $\alpha$ to render the system with the same final population size. Despite some limitations, our work has extended the research on epidemic dynamics on metapopulation networks, and therefore has given insight into epidemic spreading in a spatial population with population dynamic evolution. We point out here two problems deserving of further study: one is the empirical study on epidemic spreading model on metapopulation networks based on the real demographic and migration datasets, and another is the efficient epidemic control strategies in such networks at a lower cost.

\section{Acknowledgments}

This work was supported by the Ministry of Education Research in the Humanities and Social Sciences Planning Fund of China (Grant No. 16YJCZH023), and 2016 Jiangsu 
Overseas Research \& Training Program for University Prominent Young \& Middle-aged Teachers and President.

\section{References}

[1] R. M. Anderson, R. M. May, Infectious diseases of humans, (1991) Oxford: Oxford University press.

[2] M. E. J Newman, Spread of epidemic disease on networks, Physical Review E 64016128 (2002).

[3] R. Pastor-Satorras, A. Vespignani, Epidemic dynamics and endemic states in complex networks, Physical Review E 63066117 (2001).

[4] C. M. Joel, C. S. Anja, M. V. Erik, Edge-based compartmental modelling for infectious disease spread, Journal of the Royal Society Interface 9 890-906 (2012).

[5] J. Liindquist, J. Ma, Effective degree network disease models, Journal of Mathematical Biology 62 143-164 (2011).

[6] Y. W. Gong, Y. R. Song, G. P. Jiang, Epidemic spreading in scale-free networks including the effect of individual vigilance, Chinese Physics B 21 (1) 010205 (2012).

[7] V. Colizza, R. Pastor-Satorras, A. Vespignani, Reaction-diffusion processes and metapopulation models in heterogeneous networks, Nature Physics 3 276-282 (2007).

[8] V. Colizza, A. Vespignani, Epidemic modeling in metapopulation systems with heterogeneous coupling pattern: theory and simulations, Journal of Theoretical Biology 251 450-467 (2008).

[9] J. D. Murray, Mathematical biology ( $3^{\text {rd }}$ edition), Berlin: Springer Verlag (2005).

[10] Y. W. Gong, Y. R. Song, G. P. Jiang, Time-varying human mobility patterns with metapopulation epidemic dynamics, Physica A 392 4242-4251 (2013).

[11] S. Meloni, N. Perra, A. Arenas, S. Gómez, Y. Moreno, A. Vespignani, Modeling human mobility responses to the large-scale spreading of infectious diseases, Scientific Reports 162 (2011).

[12] A. R. North, H. Charles, J. Godfray, The dynamics of disease in a metapopulation: The role of dispersal range, Journal of Theoretical Biology, 418 57-65 (2017).

[13] H. Lund, L Lizana, I Simonsen, Effects of city-size heterogeneity on epidemic spreading in a metapopulation, Journal of Statistical Physics 151 367-382 (2013).

[14] A. S. Mata, S. C. Ferreira, R. Pastor-Satorras, Effects of local population structure in a reactiondiffusion model of a contact process on metapopulation networks, Physical Review E 88042820 (2013).

[15] Z. Ruan,M. Tang, C. Gu, J. Xu, Epidemic spreading between two coupled subpopulations with inner structures, Chaos 27103104 (2017).

[16] A. Apolloni, C. Poletto, J. J. Ramasco, P. Jensen, V. Colizza, Metapopulation epidemic models with heterogeneous mixing and travel behaviour, Theoretical Biology and Medical Modelling, 113 (2014).

[17] Y. W. Gong, Y. R. Song, G. P. Jiang, Epidemic spreading in metapopulation networks with heterogeneous infection rates, Physica A 416 208-218 (2014).

[18] J. Saldaña, Continuous-time formulation of reaction-diffusion processes on heterogeneous metapopulations, Physical Review E 78012902 (2008). 
[19] D. Juher, J. Ripoll, J. Saldaña, Analysis and Monte Carlo simulations of a model for the spread of infectious diseases in heterogeneous metapopulations, Physical Review E 80041920 (2009).

[20] B. Wang, Y. Han, G. Tanaka, Interplay between epidemic spread and information propagation on metapopulation networks, Journal of Theoretical Biology 420 18-25 (2017).

[21] S. N. Busenberg, P. van den Driessche, Analysis of a disease transmission model in a population with varying size, Journal of Theoretical Biology 28 257-270 (1990).

[22] F. Ball, D Mollison, G. Scalia-Tamba, Epidemics with two levels of mixing. The Annals of Applied Probability 7 46-89 (1997).

[23] J. Mena-Lorca J, H. W. Hethcote, Dynamic models of infectious diseases as regulators of population sizes, Journal of Theoretical Biology 30 693-716 (1992).

[24] L. Q. Linda, H. W. Hethcote, Disease transmission models with density-dependent demographics, Journal of Mathematical Biology 30 717-731 (1992).

[25] H. W. Hethcote, The mathematics of infectious diseases, SIAM Review 42 (4) 599-653 (2000).

[26] S. Bowong, J. J. Tewa, J. Kurths, Dynamics of the spread of tuberculosis in heterogeneous complex metapopulations, International Journal of Bifurcation and Chaos 231350128 (2013).

[27] J. Wang, M. Liu, Y. Li, Analysis of epidemic models with demographics in metapopulation networks, Physica A 392 1621-1630 (2013).

[28] A. Tsoularis, J. Wallace, Analysis of logistic growth models, Mathematical Bioscience 179 (1) 21-55 (2002).

[29] M. E. J. Newman, S. H. Strogatz, D. J. Watts, Random graphs with arbitrary degree distributions and their applications, Physical Review E 64026118 (2001).

[30] M. Catanzaro, M. Boguna, R. Pastor-Satorras, Generation of uncorrelated random scale-free networks, Physical Review E 71027103 (2005).

[31] J. Anderson, A Secular equation for the eigenvalues of a diagonal matrix perturbation, Linear Algebra and its Application 246 49-70 (1996).

[32] M. Keeling, P. Rohani, Modeling infectious disease in humans and animals, Princeton University Press (2008). 